\title{
Induced matchings in graphs of degree at most 4
}

\author{
Viet Hang Nguyen \\ EPFL, Switzerland \\ viethang.nguyen@epfl.ch
}

\begin{abstract}
We show that if $G$ is a connected graph of maximum degree at most 4 , which is not $C_{2,5}$, then the strong matching number of $G$ is at least $\frac{1}{9} n(G)$. This bound is tight and the proof implies a polynomial time algorithm to find an induced matching of this size.
\end{abstract}

\section{Introduction}

An induced matching of a graph $G=(V, E)$ is an edge set $M \subseteq E$ such that each vertex of $G$ is incident to at most one edge in $M$ (i.e., $M$ is a matching) and if $a b, c d \in M$ then none of the edges $a c, a d, b c, b d$ is in $E$. The maximum size of an induced matching in $G$ is called the strong matching number of $G$ and is denoted by $\nu_{s}(G)$.

While a maximum matching can be efficiently found in every graph [10], the problem of computing the strong matching number is NP-hard even in quite restricted classes. It is proved to be NP-hard for subcubic bipartite graphs [21, 4, 19], $C_{4}$-free bipartite graphs [19], line graphs [17] or cubic planar graphs [9]. In fact, even for 3-regular bipartite graphs, there is some constant $c>1$ such that the prolem cannot be approximated within a factor of $c$ unless $\mathrm{P}=\mathrm{NP}[22]$.

On the positive side, a maximum induced matching can be found efficiently in several classes of graphs such as weakly chordal graphs [6], AT-free graphs [7, graphs of bounded clique-width [17], and several other classes [2, 3, 5, 12, 13, 19].

One direction in recent research on induced matching is to lower bound the strong matching number of a graph $G$ in terms of its maximum degree $\Delta(G)$ and its order $n(G)$ or its number of edges $m(G)$. Let $G$ be a connected graph, an easy observation 22] yields

$$
\nu_{s}(G) \geq \frac{n(G)}{2\left(2 \Delta(G)^{2}-2 \Delta(G)+1\right)} .
$$

Joos [15] proved a sharp bound for $\Delta$ sufficiently large

$$
\nu_{s}(G) \geq \frac{n(G)}{\left(\left\lceil\frac{\Delta}{2}\right\rceil+1\right)\left(\left\lfloor\frac{\Delta}{2}\right\rfloor+1\right)} .
$$

He conjectured that this bound holds for all $\Delta \geq 3$ except for $G \in\left\{C_{2,5}, K_{3,3}^{+}\right\}$. Here $K_{3,3}^{+}$is the graph obtained from $K_{3,3}$ by subdividing an edge by a new vertex and $C_{2,5}$ is the graph obtained from $C_{5}$ by separating each vertex into two non adjacent vertices (see Figure 11. It is easy to see that $K_{3,3}^{+}$is a subcubic graph, $C_{2,5}$ is a 4-regular graph and $\nu_{s}\left(K_{3,3}^{+}\right)=\nu_{s}\left(C_{2,5}\right)=1$. 


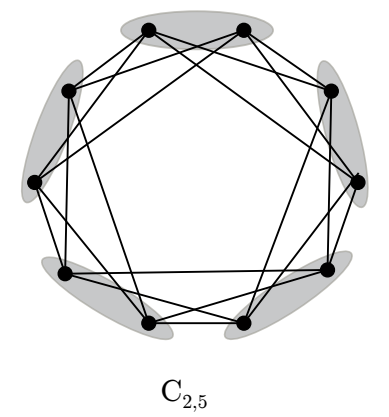

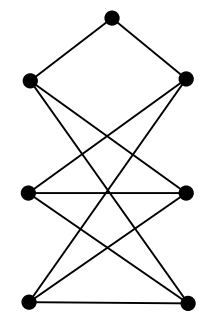

$\mathrm{K}_{3,3}^{+}$

Figure 1: $C_{2,5}$ and $K_{3,3}^{+}$

For connected subcubic graphs, Joos, Rautenbach and Sasse [15] showed that $\nu_{s}(G) \geq$ $\frac{n(G)}{6}$ if $G \neq K_{3,3}$. This result, proved by simple local reduction, strengthens an earlier lower bound $\nu_{s}(G) \geq \frac{1}{9} m(G)$ in [18] for subcubic planar graphs.

This research direction seems to be inspired by a conjecture of Erdös and Nešetřil on the strong chromatic number $\chi_{s}^{\prime}(G)$, i.e., the minimum number of induced matchings of $G$ into which $G$ can be partitioned.

Conjecture 1. If $G$ is a connected graph with maximum degree $\Delta$ then

$$
\chi_{s}^{\prime}(G) \leq \begin{cases}\frac{5}{4} \Delta^{2}, & \Delta \text { is even } ; \\ \frac{1}{4}\left(5 \Delta^{2}-2 \Delta+1\right), & \Delta \text { is odd } .\end{cases}
$$

The currently best known upper bound for the strong chromatic number is $\chi_{s}^{\prime}(G) \leq$ $1.998 \Delta^{2}$ when $\Delta$ is sufficiently large, due to Molloy and Reed [20]. Conjecture 1 is proved for subcubic graphs in [1, 14].

The conjecture of Erdős and Nešetřil, if true, implies that for a regular graph $G$ of even degree $\Delta$ we have $\nu_{s}(G) \geq \frac{2 n(G)}{5 \Delta}$, as observed in [16]. Note that Joos' conjectured bound strengthens this bound for $\Delta=4$ and the result of Joos, Rautenbach and Sasse [16] confirms Joos' conjectured bound for $\Delta=3$.

In this paper we prove the conjecture of Joos for $\Delta=4$, namely, we prove the following.

Theorem 1. Let $G \neq C_{2,5}$ be a connected graph with maximum degree at most 4 . Then the strong matching number of $G$ is at least $\frac{1}{9} n(G)$.

\section{Proof of the main theorem}

We first need some notations. For a subset $X$ of $V$ we denote by $G[X]$ the subgraph of $G$ induced by the vertices of $X$ and we use $G-X$ to denote $G[V-X]$. The number of isolated vertices of a subgraph $H$ of $G$ is denoted by $i(H)$. For $X \subset V$ we denote by $d^{\text {out }}(X)$ the number of edges between $X$ and $V-X$. When $X=\{v\}$, dout $(X)$ is simply the degree of $v$ in $G$ and is written as $d(v)$. The set of vertices adjacent to a vertex $v$ in $G$ is denoted by $N(v)$, noting that $|N(v)|=d(v)$. The minimum degree of a vertex in $G$ is denoted by $\delta(G)$.

In the remainder of this section we prove the following equivalent form of Theorem 1 
Theorem 1'. Let $G$ be a graph with maximum degree at most 4 and $G$ has no connected component which is $C_{2,5}$. Then

$$
\nu_{s}(G) \geq \frac{1}{9}(n(G)-i(G)) .
$$

We proceed by contradiction. Suppose that $G$ is a counterexample to Theorem 1' of minimum order, namely, $\Delta(G) \geq 4$,

$$
\nu_{s}(G)<\frac{1}{9} n(G),
$$

and

$$
\text { for every proper subgraph } G^{\prime} \text { of } G, \nu_{s}\left(G^{\prime}\right) \geq \frac{1}{9}\left(\left(n\left(G^{\prime}\right)-i\left(G^{\prime}\right)\right)\right. \text {. }
$$

It is worth remarking that the minimality of $G$ implies that $G$ is connected and every subgraph of $G$ is not $C_{2,5}$.

The main point of our proof is to show that $G$ satisfies

$$
G \text { has girth at least } 5 \text { and } \delta(G) \geq 3 \text {. }
$$

First, let us see how (3) implies contradiction. Suppose that $G$ satisfies (3). Let $u v$ be any edge of $G$. Then $G$ is connected by the assumption on minimality. Let $X=N(u) \cup N(v)$. Then $|X| \leq 8$ as the maximum degree of $G$ is at most 4 . Since the girth of $G$ is at least 5 , each vertex in $V(G)-X$ is adjacent to at most 2 vertices in $X$. Combining this with the assumption $\delta(G) \geq 3$ we obtain that there is no isolated vertex in $G^{\prime}=G-X$. Moreover, $G^{\prime} \neq C_{2,5}$ as remarked above. Therefore, $\nu_{s}\left(G^{\prime}\right) \geq \frac{1}{9} n\left(G^{\prime}\right)$ holds. It is easy to see that if $M$ is an induced matching of $G^{\prime}$ then $M \cup\{u v\}$ is an induced matching of $G$. Therefore,

$$
\nu_{s}(G) \geq 1=\nu_{s}\left(G^{\prime}\right)+1 \geq \frac{1}{9} n\left(G^{\prime}\right)+1 \geq \frac{1}{9}(n(G)-8)>\frac{1}{9} n(G)
$$

holds, contradicting (1).

Next, we will prove (3) through a sequence of claims using local reduction, similar to [16]. We call a vertex of degree 1 in $G$ an end-vertex. For an induced subgraph $G^{\prime}$ of $G$ we denote by $I\left(G^{\prime}\right)$ the set of isolated vertices in $G^{\prime}$ and $I_{j}\left(G^{\prime}\right) \subseteq I\left(G^{\prime}\right)$ the set of isolated vertices in $G^{\prime}$ which has degree $j$ in $G$. The cardinalities of $I\left(G^{\prime}\right), I_{j}\left(G^{\prime}\right)$ are denoted by $i\left(G^{\prime}\right), i_{j}\left(G^{\prime}\right)$, for $j \in\{1,2,3,4\}$, respectively. Then we have

$$
i\left(G^{\prime}\right)=i_{1}\left(G^{\prime}\right)+i_{2}\left(G^{\prime}\right)+i_{3}\left(G^{\prime}\right)+i_{4}\left(G^{\prime}\right) .
$$

For $X \subset V$, if $G^{\prime}=G-X$ then, since each vertex in $I\left(G^{\prime}\right)$ is adjacent only to vertices in $X$, one can see that

$$
d^{\text {out }}(X) \geq i_{1}(X)+2 i_{2}(X)+3 i_{3}(X)+4 i_{4}(X) .
$$

We also have

$$
d^{\text {out }}\left(X \cup I\left(G^{\prime}\right)\right)=d^{\text {out }}(X)-\left(i_{1}(X)+2 i_{2}(X)+3 i_{3}(X)+4 i_{4}(X)\right) .
$$

Claim 1. The neighbor of an end-vertex has degree 4. 
Proof. Let $u$ be an end-vertex and $v$ its unique neighbor. Suppose to the contrary that $d(v) \leq 3$. Let $X=\{v\} \cup N(v)$ and $G^{\prime}=G-X$. Then a simple counting shows that $|X| \leq 4$ and $d^{\text {out }}(X) \leq 6$, thus $i\left(G^{\prime}\right) \leq 6$, by (4) and (5).

If both $i\left(G^{\prime}\right)=6$ and $|X|=4$ hold, $G$ is the graph in Figure 2 and it is easy to see that $\nu_{s}(G)=2>\frac{1}{9} n(G)$, contradicting $(1)$.

If $i\left(G^{\prime}\right)<6$ or $|X|<4$, then, noting that $n\left(G^{\prime}\right)=n(G)-|X|$, we have $\nu_{s}(G) \geq$ $1+\nu_{s}\left(G^{\prime}\right) \geq 1+\frac{1}{9}\left(n\left(G^{\prime}\right)-i\left(G^{\prime}\right)\right) \geq 1+\frac{1}{9}(n(G)-9)=\frac{1}{9} n(G)$, again a contradiction to (1).

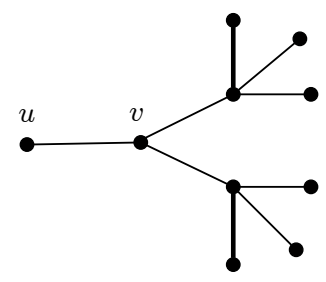

Figure 2: The graph in the proof of Claim 1 thick edges indicate the induced matching.

Claim 2. No two end-vertices have a common neighbor.

Proof. Suppose to the contrary that two end-vertices $u_{1}, u_{2}$ have a common neighbor $v$. Then $d(v)=4$ by Claim 1. Let $X=\{v\} \cup N(v)=\left\{v, u_{1}, u_{2}, w_{1}, w_{2}\right\}$ and $G^{\prime}=G-X$. Then $|X|=5$ and $d^{\text {out }}(X) \leq 6$.

If $i\left(G^{\prime}\right) \leq 4$ then $\nu_{s}(G) \geq 1+\nu_{s}\left(G^{\prime}\right) \geq 1+\frac{1}{9}\left(n\left(G^{\prime}\right)-i\left(G^{\prime}\right)\right) \geq \frac{1}{9} n(G)$, a contradiction. Therefore, let us suppose that $i\left(G^{\prime}\right) \geq 5$. Then both $w_{1}, w_{2}$ must be adjacent to some endvertices, say $t_{1}, t_{2}$, and moreover $w_{1}$ and $w_{2}$ are not adjacent, otherwise $d^{\text {out }}(X) \leq 4$, which implies $i\left(G^{\prime}\right) \leq 4$, a contradiction.

Let $X^{\prime}=X \cup N\left(w_{1}\right) \cup N\left(w_{2}\right)$ and $G^{\prime \prime}=G-X^{\prime}$. Then $\left|X^{\prime}\right| \leq 11$ and $d^{\text {out }}\left(X^{\prime}\right) \leq 3$, which implies $i\left(G^{\prime \prime}\right) \leq 3$ (see Figure 3). Since for every induced matching $M$ of $G^{\prime \prime}$, $M \cup\left\{w_{1} t_{1}, w_{2} t_{2}\right\}$ is an induced matching of $G$, we have

$$
\nu_{s}(G) \geq 2+\nu_{s}\left(G^{\prime \prime}\right) \geq 2+\frac{1}{9}\left(n\left(G^{\prime \prime}\right)-i\left(G^{\prime \prime}\right)\right) \geq 2+\frac{1}{9}(n(G)-11-3)>\frac{1}{9} n(G),
$$

a contradiction.

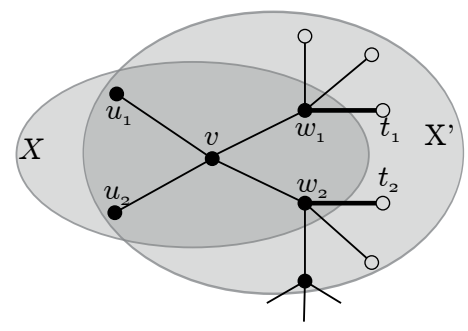

Figure 3: An illustration for the proof of Claim 2

Claim 3. No two end-vertices have distance 4 in $G$. 
Proof. Suppose to the contrary that two end-vertices $u_{1}$ and $u_{2}$ have distance 4 in $G$ and $u_{1} v_{1} w v_{2} u_{2}$ is a path of length 4 linking $u_{1}$ and $u_{2}$. Consider $X=\left\{v_{1}, v_{2}\right\} \cup N\left(v_{1}\right) \cup N\left(v_{2}\right)$. Then $|X| \leq 9$, and $d^{\text {out }}(X) \leq 14$. From (4) and (5) we have $d^{\text {out }}(X) \geq 2 i\left(G^{\prime}\right)-i_{1}\left(G^{\prime}\right)$. Thus, if $i\left(G^{\prime}\right) \geq 10$ then $i_{1}\left(G^{\prime}\right) \geq 2 i\left(G^{\prime}\right)-d^{\text {out }}(X) \geq 6$. However, vertices in $I_{1}\left(G^{\prime}\right)$ are only adjacent to vertices in set $X-\left\{v_{1}, v_{2}, u_{1}, v_{1}\right\}$, which consists of at most 5 vertices. Hence, there are two end-vertices in $I_{1}\left(G^{\prime}\right)$ that have a common neighbor, contradicting Claim 2 . Therefore, $i\left(G^{\prime}\right) \leq 9$, and, noting that each induced matching $M$ of $G^{\prime}$ can be extended to an induced matching $M \cup\left\{u_{1} v_{1}, u_{2} v_{2}\right\}$ of $G$, we derive that

$$
\nu_{s}(G) \geq 2+\nu_{s}\left(G^{\prime}\right) \geq 2+\frac{1}{9}\left(n\left(G^{\prime}\right)-i\left(G^{\prime}\right)\right) \geq 2+\frac{1}{9}(n(G)-9-9)=\frac{1}{9} n(G),
$$

a contradiction to (1). (See Figure 4.)

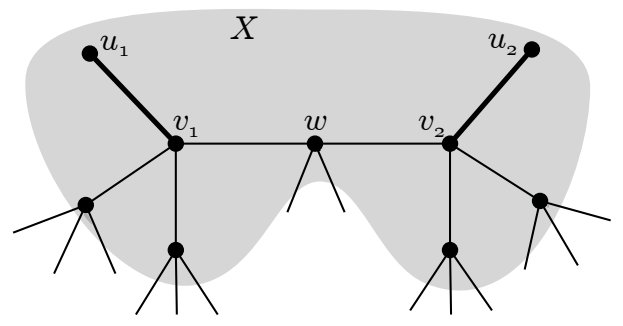

Figure 4: An illustration for the proof of Claim 3

Claim 4. $\delta(G) \geq 2$.

Proof. Suppose to the contrary that $u$ is a vertex of degree 1 and $v$ is its unique neighbor in $G$. Then, by Claim 1, $v$ has 3 other neighbors $w_{1}, w_{2}, w_{3}$. Let $X=\left\{u, v, w_{1}, w_{2}, w_{3}\right\}$ and $G^{\prime}=G-X$. Since each induced matching $M$ of $G^{\prime}$ can be extended to an induced matching $M \cup\{u v\}$ of $G$, if $i\left(G^{\prime}\right) \leq 4$ then

$$
\nu_{s}(G) \geq 1+\nu_{s}\left(G^{\prime}\right) \geq 1+\frac{1}{9}\left(n\left(G^{\prime}\right)-i\left(G^{\prime}\right)\right) \geq 1+\frac{1}{9}(n(G)-5-4)=\frac{1}{9} n(G)
$$

holds, contradicting (1). Therefore, let us suppose that $i\left(G^{\prime}\right) \geq 5$. Since

$$
2 i\left(G^{\prime}\right)-i_{1}\left(G^{\prime}\right) \leq d^{\text {out }}(X) \leq 9,
$$

we must have $i_{1}\left(G^{\prime}\right) \geq 1$. By Claim 2 and 3 , if $s$ and $t$ are two vertices in $I_{1}\left(G^{\prime}\right)$ then they are adjacent to two distinct vertices $w_{i}$ and $w_{j}$ and furthermore, $w_{i}$ and $w_{j}$ are adjacent. Hence,

$$
d^{\text {out }}(X) \leq 9-2\left(i_{1}\left(G^{\prime}\right)-1\right)=11-2 i_{1}\left(G^{\prime}\right) .
$$

Therefore, by (5), $11 \geq 3 i_{1}\left(G^{\prime}\right)+2 i_{2}\left(G^{\prime}\right)+3 i_{3}\left(g^{\prime}\right)+4 i_{4}\left(G^{\prime}\right)$ holds, which, together with $i_{1}\left(G^{\prime}\right) \geq 1$ and $i\left(G^{\prime}\right) \geq 5$, implies that $i_{1}\left(G^{\prime}\right)=1, i_{2}\left(G^{\prime}\right)=4, i_{3}\left(G^{\prime}\right)=i_{4}\left(G^{\prime}\right)=0$ and furthermore $w_{1}, w_{2}, w_{3}$ all have degree 4 and are not adjacent. But then $d^{\text {out }}\left(X \cup I\left(G^{\prime}\right)\right)=0$ and hence $n(G)=10$. Let $s$ be the unique vertex in $I_{1}\left(G^{\prime}\right)$ and suppose without loss of generality that its unique neighbor in $G$ is $w_{1}$. Since $d\left(w_{1}\right)=4$ and $i_{2}\left(G^{\prime}\right)=4$, there is a vertex $t$ in $I_{2}\left(G^{\prime}\right)$ that is not adjacent to $w_{1}$. Then, $\left\{w_{1} s, w_{2} t\right\}$ is an induced matching of $G$, and thus $\nu_{s}(G) \geq 2>\frac{1}{9} n(G)$, a contradiction. 
Claim 5. No two vertices of degree 2 are adjacent.

Proof. Suppose to the contrary that $u, v$ are two adjacent vertices of degree 2. Let $X=$ $N(u) \cup N(v)$ and $G^{\prime}=G-X$. Then $|X|=4$ and $d^{\text {out }}(X) \leq 6$. Remembering that $i_{1}(G)=0$ by Claim 4 , we see that $i\left(G^{\prime}\right) \leq\left\lfloor\frac{1}{2} d^{\text {out }}(X)\right\rfloor \leq 3$ by $(5)$. Since for each induced matching $M$ of $G^{\prime}, M \cup\{u v\}$ is an induced matching of $G$, it follows that

$$
\nu_{s}(G) \geq 1+\nu_{s}\left(G^{\prime}\right) \geq 1+\frac{1}{9}\left(n\left(G^{\prime}\right)-i\left(G^{\prime}\right)\right) \geq 1+\frac{1}{9}(n(G)-4-3) \geq \frac{1}{9} n(G)
$$

holds, a contradiction.

Claim 6. No vertex of degree 2 is contained in a triangle.

Proof. Suppose to the contrary that $u$ is a vertex of degree 2 which is contained in a triangle and $v$ is one of its neighbors. Let $X=N(u) \cup N(v)$ and $G^{\prime}=G-X$. Then $|X| \leq 5$ and $d^{\text {out }}(X) \leq 8$. On the other hand, by Claim 4 and $(5)$, dout $(X) \geq 2 i_{2}\left(G^{\prime}\right)+3 i_{3}\left(G^{\prime}\right)+4 i_{4}\left(G^{\prime}\right)$ and $i\left(G^{\prime}\right)=i_{2}\left(G^{\prime}\right)+i_{3}\left(G^{\prime}\right)+i_{4}\left(G^{\prime}\right)$ hold. Hence $i\left(G^{\prime}\right) \leq d^{\text {out }}(X) / 2 \leq 4$. Therefore,

$$
\nu_{s}(G) \geq 1+\nu_{s}\left(G^{\prime}\right) \geq 1+\frac{1}{9}(n(G)-5-4)=\frac{1}{9} n(G),
$$

a contradiction.

Claim 7. No vertex of degree 2 is contained in a cycle of length 4.

Proof. Suppose that $u$ is a vertex of degree 2 which is contained in a cycle uvwt of length 4. Let $X=N(u) \cup N(v)$ and $G^{\prime}=G-X$. Then $|X| \leq 6$ and $d^{\text {out }}(X) \leq 10$, thus $i\left(G^{\prime}\right) \leq d^{\text {out }}(X) / 2 \leq 5$.

If $i\left(G^{\prime}\right) \leq 3$ then $\nu_{s}(G) \geq 1+\nu_{s}\left(G^{\prime}\right) \geq \frac{1}{9} n(G)$, contradicting (1). Hence we may suppose that $i\left(G^{\prime}\right) \geq 4$. Then there exists a vertex $s$ in $I\left(G^{\prime}\right)$ that is not adjacent to $t$. Since $\delta(s) \geq 2$ by Claim 4 $s$ is adjacent to a vertex $r$ in $N(v)-\{u, w\}$. Also we have $\left|N\left(X \cup I\left(G^{\prime}\right)\right)\right| \leq d^{\text {out }}\left(X \cup I\left(G^{\prime}\right)\right) \leq d^{\text {out }}(X)-2 i\left(G^{\prime}\right) \leq 2$, where the second inequality follows from (4), (5), (6) and Claim 4 .

Now let $X^{\prime}=X \cup I\left(G^{\prime}\right) \cup N\left(X \cup I\left(G^{\prime}\right)\right)$ and $G^{\prime \prime}=G-X^{\prime}$. Then $\left|X^{\prime}\right| \leq|X|+i\left(G^{\prime}\right)+$ $\left|N\left(X \cup I\left(G^{\prime}\right)\right)\right| \leq 6+5+2=13$. Moreover, $d^{\text {out }}\left(X^{\prime}\right) \leq 3\left|N\left(X \cup I\left(G^{\prime}\right)\right)\right| \leq 6$. Hence $i\left(G^{\prime \prime}\right) \leq d^{\text {out }}\left(X^{\prime}\right) / 2 \leq 3$. Since for each induced matching $M$ of $G^{\prime \prime}, M \cup\{u t, r s\}$ is an induced matching of $G^{\prime \prime}$, it follows that

$$
\nu_{s}(G) \geq 2+\nu_{s}\left(G^{\prime \prime}\right) \geq \frac{1}{9} n(G),
$$

contradicting (1). (see Figure 5)

Claim 8. $\delta(G) \geq 3$.

Proof. Suppose to the contrary that $u$ is a vertex of degree 2 in $G$ and $v, w$ are its neighbors. Let $X=N(u) \cup N(v)$ and $G^{\prime}=G-X$. Then $|X| \leq 6$ and $d^{o u t}(X) \leq 12$. By Claim 7, no vertex of degree 2 is contained in a cycle of length 4 , thus $w$ is not adjacent to any neighbor of $v$ other than $u$. Now if $i\left(G^{\prime}\right) \leq 3$ then $\nu_{s}(G) \geq 1+\nu_{s}\left(G^{\prime}\right) \geq 1+\frac{1}{9}(n(G)-6-3)=\frac{1}{9} n(G)$, a contradiction.

So let us suppose that $i\left(G^{\prime}\right) \geq 4$. Then there is a vertex $s \in I\left(G^{\prime}\right)$ that is not adjacent to $w$, note that then $s$ is adjacent only to vertices in $N(v)-\{u\}$, so $s$ is contained in a cycle 


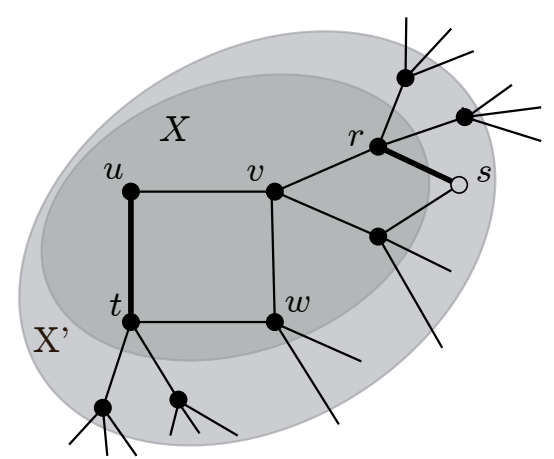

Figure 5: An illustration for the proof of Claim 7.

of length 4. Therefore $d(s) \geq 3$ by Claim 7. Let $t \in N(v)$ be one of its neighbors. Then $\{s t, u w\}$ is an induced matching of $G$ (see Figure 6).

Since vertices in $I\left(G^{\prime}\right)$ are adjacent only to vertices in $X$ we have $d^{\text {out }}\left(X \cup I\left(G^{\prime}\right)\right) \leq$ $d^{\text {out }}(X)-\sum_{x \in I\left(G^{\prime}\right)} d(x)$. Since $d(x) \geq 2$ for all $x \in I\left(G^{\prime}\right)$, by Claim 4 , $d(s) \geq 3$ and $i\left(G^{\prime}\right) \geq 4$, we have $\sum_{x \in I\left(G^{\prime}\right)} d(x) \geq 3 \times 2+3=9$. Thus $d^{\text {out }}\left(X \cup I\left(G^{\prime}\right)\right) \leq 3$.

Let $X^{\prime}=X \cup I\left(G^{\prime}\right) \cup N\left(X \cup I\left(G^{\prime}\right)\right)$ and $G^{\prime \prime}=G-X^{\prime}$. Then one can easily see that $d^{\text {out }}\left(X^{\prime}\right) \leq 3\left|N\left(X \cup I\left(G^{\prime}\right)\right)\right| \leq 3 d^{\text {out }}\left(X \cup I\left(G^{\prime}\right)\right) \leq 9$, so $i\left(G^{\prime \prime}\right) \leq\left\lfloor\frac{d^{\text {out }}\left(X^{\prime}\right)}{2}\right\rfloor=4$, by Claim 4 and (5). Now let us upper bound $\left|X^{\prime}\right|$. We have

$$
\begin{aligned}
\left|X^{\prime}\right| & \leq|X|+i\left(G^{\prime}\right)+d^{\text {out }}\left(X \cup I\left(G^{\prime}\right)\right. \\
& \leq|X|+i\left(G^{\prime}\right)+d^{\text {out }}(X)-2 i\left(G^{\prime}\right) \\
& =|X|+d^{\text {out }}(X)-i\left(G^{\prime}\right) \\
& \leq 6+12-4 \\
& =14 .
\end{aligned}
$$

Noting that every matching $M$ of $G^{\prime \prime}$ can be extended to a matching $M \cup\{s t, u w\}$ of $G$, we derive

$$
\nu_{s}(G) \geq 2+\nu_{s}\left(G^{\prime \prime}\right) \geq 2+\frac{1}{9}(n(G)-14-4)=\frac{1}{9} n(G),
$$

a contradiction.

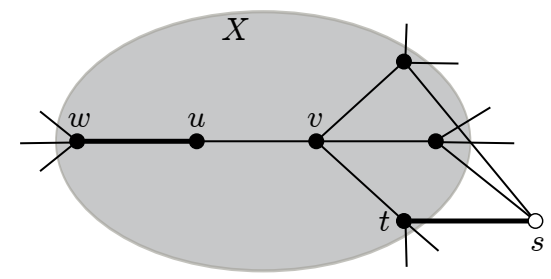

Figure 6: An illustration for the proof of Claim 8

Claim 9. $G$ contains no triangle.

Proof. Suppose to the contrary that uvw is a triangle in $G$. Let $X=N(u) \cup N(v)$ and $G^{\prime}=G-X$. Then $|X| \leq 7$. So if $i\left(G^{\prime}\right) \leq 2$ we have $\nu_{s}(G) \geq 1+\nu_{s}\left(G^{\prime}\right) \geq 1+\frac{1}{9}(n(G)-7-2)=$ $\frac{1}{9} n(G)$, a contradiction. 
Let us suppose that $i\left(G^{\prime}\right) \geq 3$. It is easy to see that $d^{\text {out }}(X) \leq 14$. Hence, by Claim 8 and (5), $i\left(G^{\prime}\right)=i_{3}\left(G^{\prime}\right)+i_{4}\left(G^{\prime}\right) \leq\left\lfloor d^{\text {out }}(X) / 3\right\rfloor \leq 4$. Since $|N(w)-X| \leq 2$, and $\delta(G) \geq 3$ by Claim 8, there exists a vertex $s \in I\left(G^{\prime}\right)$ such that $s$ is not adjacent to $w$. Then $s$ is adjacent to a vertex $r \in N(v)-\{u, w\}$ and $\{u w, s r\}$ is an induced matching of $G$.

Let $X^{\prime}=X \cup I\left(G^{\prime}\right) \cup N(w) \cup N(r)$ and $G^{\prime \prime}=G-X^{\prime}$. Let $Y=(N(w) \cup N(r))-\left(X \cup I\left(G^{\prime}\right)\right.$. Then by simple counting one can see that $|Y| \leq 4$ and $\left|X^{\prime}\right|=|X|+i\left(G^{\prime}\right)+|Y| \leq 11+i\left(G^{\prime}\right)$.

Since each vertex in $Y$ is adjacent to at least one vertex in $X$ and to at most 3 vertices outside $X$, we have

$$
\begin{aligned}
d^{\text {out }}\left(X^{\prime}\right) & \leq d^{\text {out }}\left(X \cup I\left(G^{\prime}\right)\right)-|Y|+3|Y| \\
& \leq d^{\text {out }}(X)-3 i\left(G^{\prime}\right)+2|Y|,
\end{aligned}
$$

where the last equality follows from 6 and Claim 8 . Therefore,

$$
\begin{aligned}
\left|X^{\prime}\right|+i\left(G^{\prime \prime}\right) & \leq 11+i\left(G^{\prime}\right)+\left\lfloor\frac{d^{\text {out }}\left(X^{\prime}\right)}{3}\right\rfloor \\
& \leq 11+\left\lfloor\frac{d^{\text {out }}(X)+2|Y|}{3}\right\rfloor \\
& \leq 11+\left\lfloor\frac{14+2 \times 4}{3}\right\rfloor \\
& =18 .
\end{aligned}
$$

Since each induced matching $M$ of $G^{\prime \prime}$ can be extended to an induced matching $M \cup\{u w, s r\}$ of $G$, it follows that

$$
\nu_{s}(G) \geq 2+\nu_{s}\left(G^{\prime \prime}\right) \geq 2+\frac{1}{9}(n(G)-18)=\frac{1}{9} n(G),
$$

holds, contradicting (1).

Claim 10. If a vertex $u$ is contained in a cycle of length 4 then $d(u) \geq 4$.

Proof. Suppose to the contrary that $u$ is contained in a cycle $C=u v x y$ of length 4 and $d(u)=3$. Let $v$ be the neighbor of $u$ that is not contained in $C$. Let $X=N(u) \cup N(v)$ and $G^{\prime}=G-X$. Then $|X| \leq 7$ and $d^{\text {out }}(X) \leq 13$. Hence, if $i\left(G^{\prime}\right) \leq 2$ then $\nu_{s}(G) \geq$ $1+\nu_{s}\left(G^{\prime}\right) \geq \frac{1}{9} n(G)$ holds, a contradiction. So let us suppose that $i\left(G^{\prime}\right) \geq 3$.

If $d(v)=3$ then $|X|=6$ and $d^{\text {out }}(X) \leq 10$, thus $i\left(G^{\prime}\right) \leq 3$. Hence $\nu_{s}(G) \geq 1+$ $\nu_{s}\left(G^{\prime}\right) \geq \frac{1}{9} n(G)$ holds, again a contradiction. So we may suppose that $d(v)=4$ and $N(v)=\{u, x, t, r\}$.

We will use the following assertion.

Assertion 1. If there is an induced matching in $G\left[X \cup I\left(G^{\prime}\right)\right]$ then $\nu_{s}(G) \geq \frac{1}{9} n(G)$.

Proof. Let $\{a b, c d\}$ be an induced matching in $G\left[X \cup I\left(G^{\prime}\right)\right]$. Let $X_{1}=X \cup I\left(G^{\prime}\right) \cup$ $N(\{a, b, c, d\})$ and $Y_{1}=N(\{a, b, c, d\})-\left(X \cup I\left(G^{\prime}\right)\right.$. Then since each vertex in $Y_{1}$ is adjacent to a vertex in $\{a, b, c, d\} \subset X \cup I\left(G^{\prime}\right)$ and $\delta(G) \geq 3$ by Claim 8, we have $\left|Y_{1}\right| \leq$ $d^{\text {out }}\left(X \cup I\left(G^{\prime}\right)\right)-3 i\left(G^{\prime}\right) \leq 13-3 \times 3=4$. Using a similar argument as in the proof of Claim 9, we obtain

$$
d^{\text {out }}\left(X_{1}\right) \leq d^{\text {out }}(X)-3 i\left(G^{\prime}\right)-\left|Y_{1}\right|+3\left|Y_{1}\right|=d^{\text {out }}(X)-3 i\left(G^{\prime}\right)+2\left|Y_{1}\right|,
$$


and

$$
\left|X_{1}\right|=|X|+\left|I\left(G^{\prime}\right)\right|+\left|Y_{1}\right|
$$

Therefore,

$$
\begin{aligned}
\left|X_{1}\right|+i\left(G_{1}\right) & \leq|X|+i\left(G^{\prime}\right)+\left|Y_{1}\right|+\frac{d^{\text {out }}\left(X_{1}\right)}{3} \\
& =|X|+\frac{5}{3}\left|Y_{1}\right|+\frac{d^{\text {out }}(X)}{3} \\
& \leq 7+\frac{5}{3} \times 4+\frac{13}{3} \\
& =18 .
\end{aligned}
$$

Hence, since for each induced matching $M$ of $G^{\prime \prime}, M \cup\{a b, c d\}$ is an induced matching of $G$, we have

$$
\nu_{s}(G) \geq 2+\nu_{s}\left(G_{1}\right) \geq 2+\frac{1}{9}(n(G)-18)=\frac{1}{9} n(G),
$$

a contradiction.

Now we are ready to complete the proof of Claim 10. Since $i\left(G^{\prime}\right) \geq 3$ and $\mid N(t)-$ $X|| N,(r)-X \mid \leq 2$, by observing that each vertex in $I\left(G^{\prime}\right)$ must be adjacent to either $r$ or $t$, we deduce that there are two vertices $s_{1}, s_{2} \in I\left(G^{\prime}\right)$ such that $s_{1}$ is adjacent to $r$ but not to $t$ and $s_{2}$ is adjacent to $t$ but not to $r$. Then since $r$ and $t$ are not adjacent by Claim 9 , $\left\{s_{1} r, s_{2} t\right\}$ is an induced matching of $G\left[X \cup I\left(G^{\prime}\right)\right]$. So Assertion 1 implies contradiction.

Claim 11. There is no cycle of length 4.

Proof. Suppose that $C=u v x y$ is a cycle of length 4 in $G$. Let $X=N(u) \cup N(v)$ and $G^{\prime}=G-X$. Then $|X| \leq 8$ and $d^{\text {out }}(X) \leq 16$. If $i\left(G^{\prime}\right) \leq 1$ then a similar argument as in previous claims yields $\nu_{s}(G) \geq \frac{1}{9} n(G)$, a contradiction. Therefore, we may suppose that $i\left(G^{\prime}\right) \geq 2$.

Since there are no three vertices in $X$ with pairwise distance 3 and each vertex in $I\left(G^{\prime}\right)$ has degree at least 3 we obtain that each vertex of $I\left(G^{\prime}\right)$ lies on a cycle of length 4 . Therefore, by Claim 10 we have

$$
\text { all vertices in } I\left(G^{\prime}\right) \text { have degree } 4 \text {. }
$$

We will use the following assertion, which is similar to Assertion 1 .

Assertion 2. If there is an induced matching in $G\left[X \cup I\left(G^{\prime}\right)\right]$ then $\nu_{s}(G) \geq \frac{1}{9} n(G)$.

Proof. Let $\{a b, c d\}$ be an induced matching in $G\left[X \cup I\left(G^{\prime}\right)\right]$. Let $X_{1}=X \cup I\left(G^{\prime}\right) \cup$ $N(\{a, b, c, d\})$ and $Y_{1}=N(\{a, b, c, d\})-\left(X \cup I\left(G^{\prime}\right)\right)$.

We first prove that if $\left|Y_{1}\right| \leq 4$ then $\nu_{s}(G) \geq \frac{1}{9} n(G)$, a contradiction. In fact, a similar argument as in the proof of Assertion 1 yields

$$
d^{\text {out }}\left(X_{1}\right) \leq d^{\text {out }}(X)-4 i\left(G^{\prime}\right)-\left|Y_{1}\right|+3\left|Y_{1}\right|=d^{\text {out }}(X)-4 i\left(G^{\prime}\right)+2\left|Y_{1}\right|,
$$

where the multiplicity 4 for $i\left(G^{\prime}\right)$ is due to $(7)$. We also have

$$
\left|X_{1}\right|=|X|+\left|I\left(G^{\prime}\right)\right|+\left|Y_{1}\right| .
$$


Therefore,

$$
\begin{aligned}
\left|X_{1}\right|+i\left(G_{1}\right) & \leq|X|+i\left(G^{\prime}\right)+|Y|+\frac{d^{\text {out }}\left(X_{1}\right)}{4} \\
& =|X|+\frac{3}{2}\left|Y_{1}\right|+\frac{d^{\text {out }}(X)}{4} \\
& \leq 8+\frac{3}{2} \times 4+\frac{16}{4} \\
& =18 .
\end{aligned}
$$

It follows that

$$
\nu_{s}(G) \geq 2+\nu_{s}\left(G_{1}\right) \geq 2+\frac{1}{9}(n(G)-18)=\frac{1}{9} n(G)
$$

holds.

It remains to prove that $\left|Y_{1}\right| \leq 4$. Indeed, if $\left|\{a, b, c, d\} \cap I\left(G^{\prime}\right)\right| \geq 2$, then it is easy to see that $\left|Y_{1}\right|=\mid N\left(\{a, b, c, d\}-\left(X \cup I\left(G^{\prime}\right)\right) \mid \leq 4\right.$. On the other hand, if $\{a, b, c, d\} \subset X$ then a simple counting shows that $d^{\text {out }}(X) \leq 12$ and hence $\left|Y_{1}\right| \leq d^{\text {out }}(X)-4 i\left(G^{\prime}\right) \leq 4$, where the coefficient 4 of $i\left(G^{\prime}\right)$ is due to (7). Thus we may suppose that $a \in I\left(G^{\prime}\right)$ and $\{b, c, d\} \subset X$, note that then $b \notin\{u, v\}$. Let $t \in I\left(G^{\prime}\right)-s$. First consider the case $\{c, d\} \cap\{u, v\}=\emptyset$. Since $d(s)=d(t)=4$ and $|X-\{u, v, b, c, d\}|=3$ we have that both $s$ and $t$ have a neighbor in $\{b, c, d\}$. This implies that $\left|Y_{1}\right|=\mid N(\{a, b, c, d\})-\left(X \cup I\left(G^{\prime}\right) \mid \leq 4\right.$, as desired. Next, consider the case $\{c, d\}=\{u, v\}$, it is easy to see that then $\left|Y_{1}\right| \leq 2$. Finally, consider the case $|\{c, d\} \cap\{u, v\}|=1$, say $d \in\{u, v\}$ and $c \notin\{u, v\}$. Then since $|N(t) \cap\{x, y\}| \leq 1$, as $G$ contains no triangle, $d(t)=4$ and $|X-\{u, v, x, y\}|=4$, we have that $t$ must be adjacent to $b$ or $c$. Hence again we obtain $|Y-1| \leq 4$, as desired.

Now suppose that there is no induced matching of size 2 in $G\left[X \cup I\left(G^{\prime}\right)\right]$. We prove that $G=C_{2,5}$. Let $N(v)=\{u, x, a, b\}$ and $N(u)=\{v, y, c, d\}$. Since each vertex $s$ in $I\left(G^{\prime}\right)$ is not adjacent to both $x$ and $y$ at the same time as $G$ has no triangle, we may assume without loss of generality that there is an $s \in I\left(G^{\prime}\right)$ that is adjacent to $a, c$ but not to $y$. Then since $\{s a, y u\}$ is not an induced matching of $G\left[X \cup I\left(G^{\prime}\right)\right]$ and since $u a \notin E$ by Claim 9 , we must have $y a \in E$. (See Figure 7 for an illustration.) Now by considering pair $\{d u, s a\}$, since $u a, u s \notin E$, we see that either $d a \in E$ or $s d \in E$, but not both of them belong to $E$.
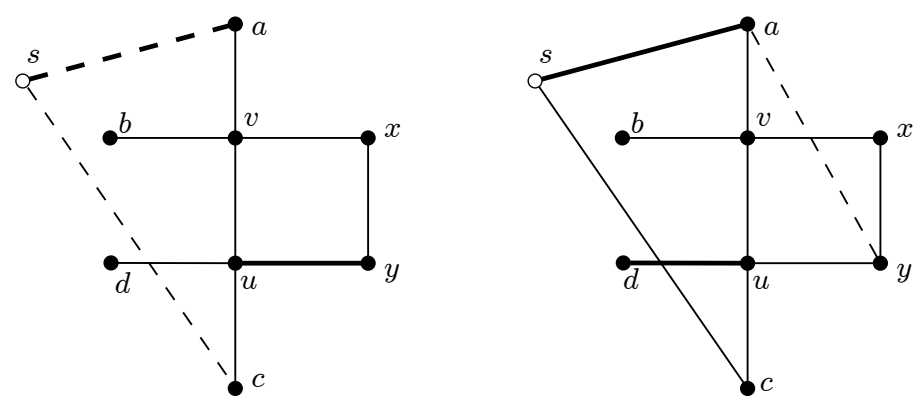

Figure 7: An illustration of Case 1; dashed edges indicate newly "found" edges and thick edges indicate considered pair in each step.

Case 1: $d a \in E$ and $s d \notin E$.

(See an illustration for this case in Figure 8)

Since $d(s)=4$ we derive that $s x, s b \in E$. Consider pair $\{s x, u d\}$, we see that $d x \in E$ since $u x, u s, u d \notin E$. Now let $t$ be a vertex in $I\left(G^{\prime}\right)-\{s\} \neq \emptyset$, due to the assumption that 

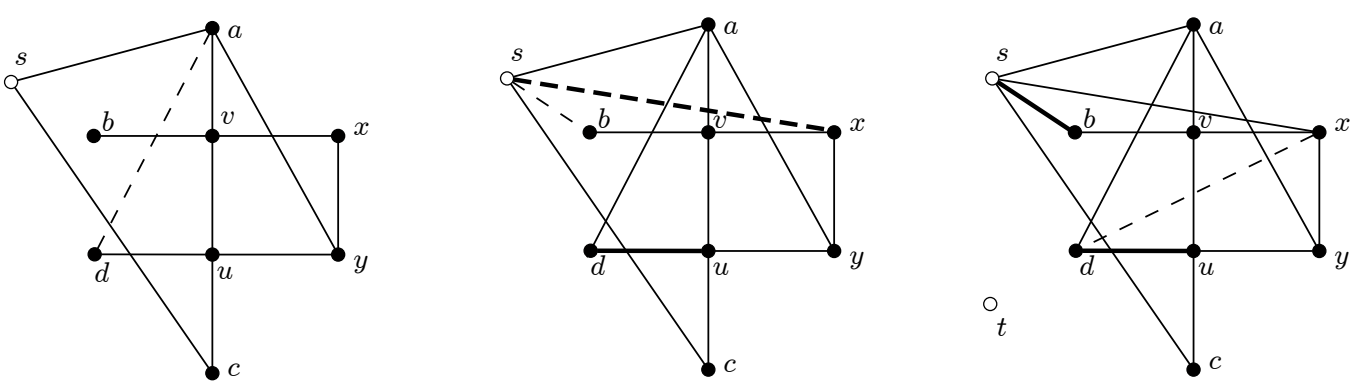

Figure 8: An illustration for Case 1; dashed edges indicate newly "found" edge and thick edges indicate considered pair in each step.

$I\left(G^{\prime}\right) \geq 2$. Then since $d(a)=d(x)=4$, we know that $N(a)=\{s, t, x, y\}, N(x)=\{b, d, y, v\}$ and $t a \notin E$. Therefore, $N(t) \subseteq\{b, c, d, x, y\}$ holds. However, by considering pair $\{s b, u d\}$ we derive that $b d \in E$ which implies that $G$ contains a triangle $t b d$, contradicting Claim 9 .

Case 2: $s d \in E$ and $d a \notin E$.

Consider pair $\{s c, b v\}$, using the fact that $G$ contains no triangle from Claim 9, we see that either $b s$ or $b c$ is in $E$ but not both of them. Hence we consider these cases separately.

Case 2.1: $b c \in E$ and $b s \notin E$.

(See Figure 9 for an illustration).

Then, since $d(s)=4$ and $s y \notin E$, we have that $s x \in E$. Also we have $a c \notin E$, otherwise asc is a triangle in $G$, contradicting Claim 9. By considering pair $\{b c, a y\}$ we obtain that $b y \in E$. Now let $t \in I\left(G^{\prime}\right)-\{s\} \neq \emptyset$. Since $N(y)=\{x, u, a, b\}$ and $t b, t c$ do not both belong to $E$, we must have $t x, t d, t a \in E$. Considering pair $\{u c, t x\}$, noting that $x c \notin E$ since otherwise $s c x$ is a triangle in $G$, we derive that $t c \in E$. Finally, by considering pair $\{b y, s d\}$, we obtain that $b d \in E$. However then $V(G)=X$ and $G=C_{2,5}$, where the pairs corresponding to the vertices of $C_{5}$ are $\{a, x\},\{v, y\},\{b, u\},\{c, d\},\{s, t\}$, a contradiction to the assumption that $G \neq C_{2,5}$.

Case 2.2: $b s \in E$ and $b c \notin E$.

(See Figure 10 for an illustration.)

Then considering pair $\{s b, u y\}$ we derive that $y b \in E$. Now let $t \in I\left(G^{\prime}\right)-\{s\}$, then we may assume without loss of generality that $t a, t c \in E$. Considering pair $\{t c, b y\}$, we have that $t b \in E$. Considering pair $\{t a, u d\}$, we obtain that $t d \in E$. By considering pair $\{x y, t c\}$ we deduce that $x c \in E$. Finally, by considering pair $\{t d, x v\}$ we derive that $x d \in E$. Therefore, $V(G)=X \cup I\left(G^{\prime}\right)$ and $G=C_{2,5}$ where the pairs corresponding to the vertices of $C_{5}$ are $\{x, u\},\{v, y\},\{a, b\}\{c, d\},\{s, t\}$, a contradiction to the assumption that $G \neq C_{2,5}$.

The example in Figure 11, due to Joos [15], shows that the lower bound in Theorem 1 is tight. It is easy to see that the proof of Theorem 1 implies a polynomial time algorithm to find an induced matching of size $\frac{1}{9} n(G)$.

\section{References}

[1] L. D. Andersen, The strong chromatic index of a cubic graph is at most 10, Discrete Math. 108 (1992), no. 1-3, 231-252. 

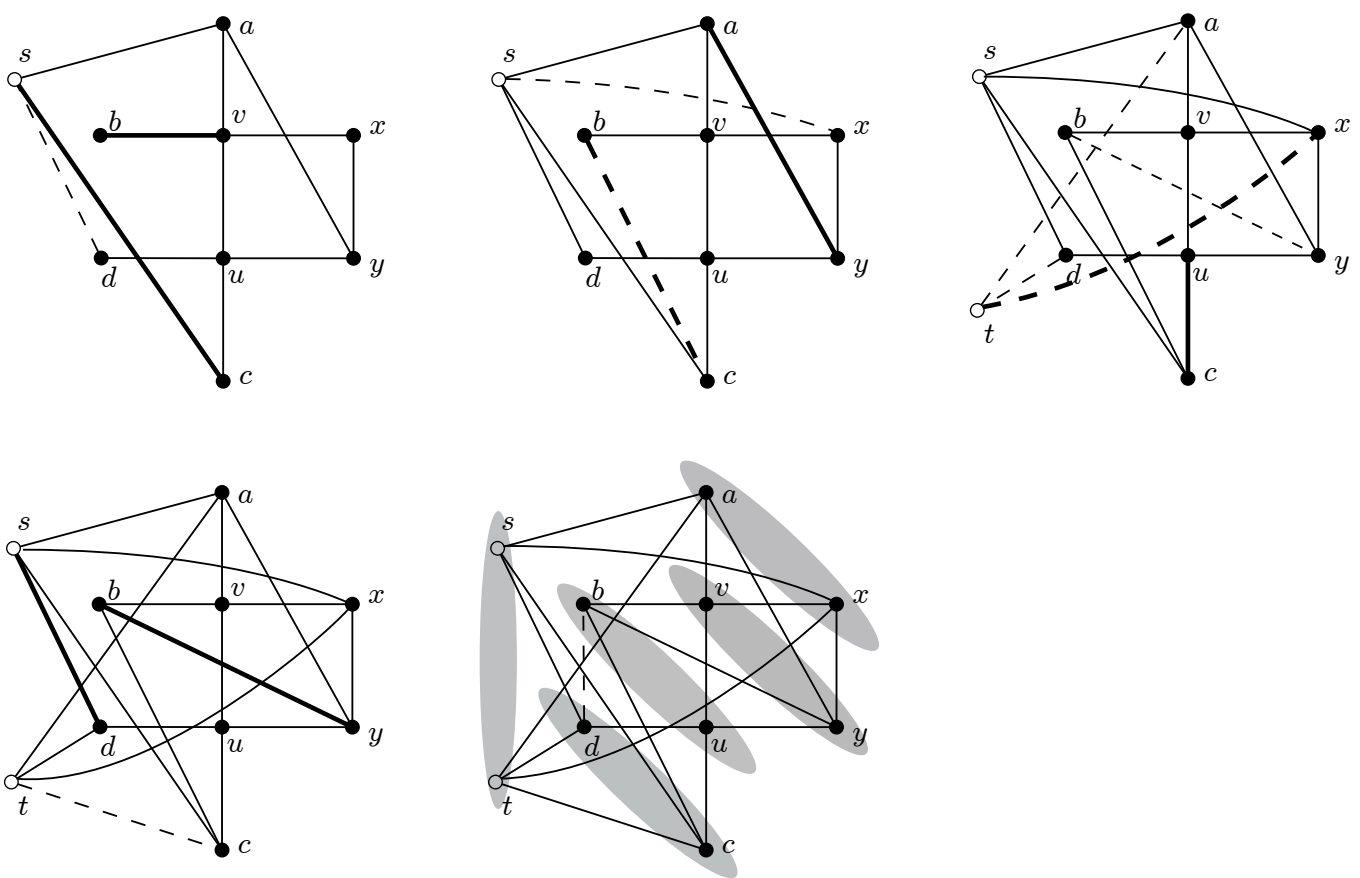

Figure 9: An illustration for Case 2.1; dashed edges indicate newly "found" edge and thick edges indicate considered pair in each step.
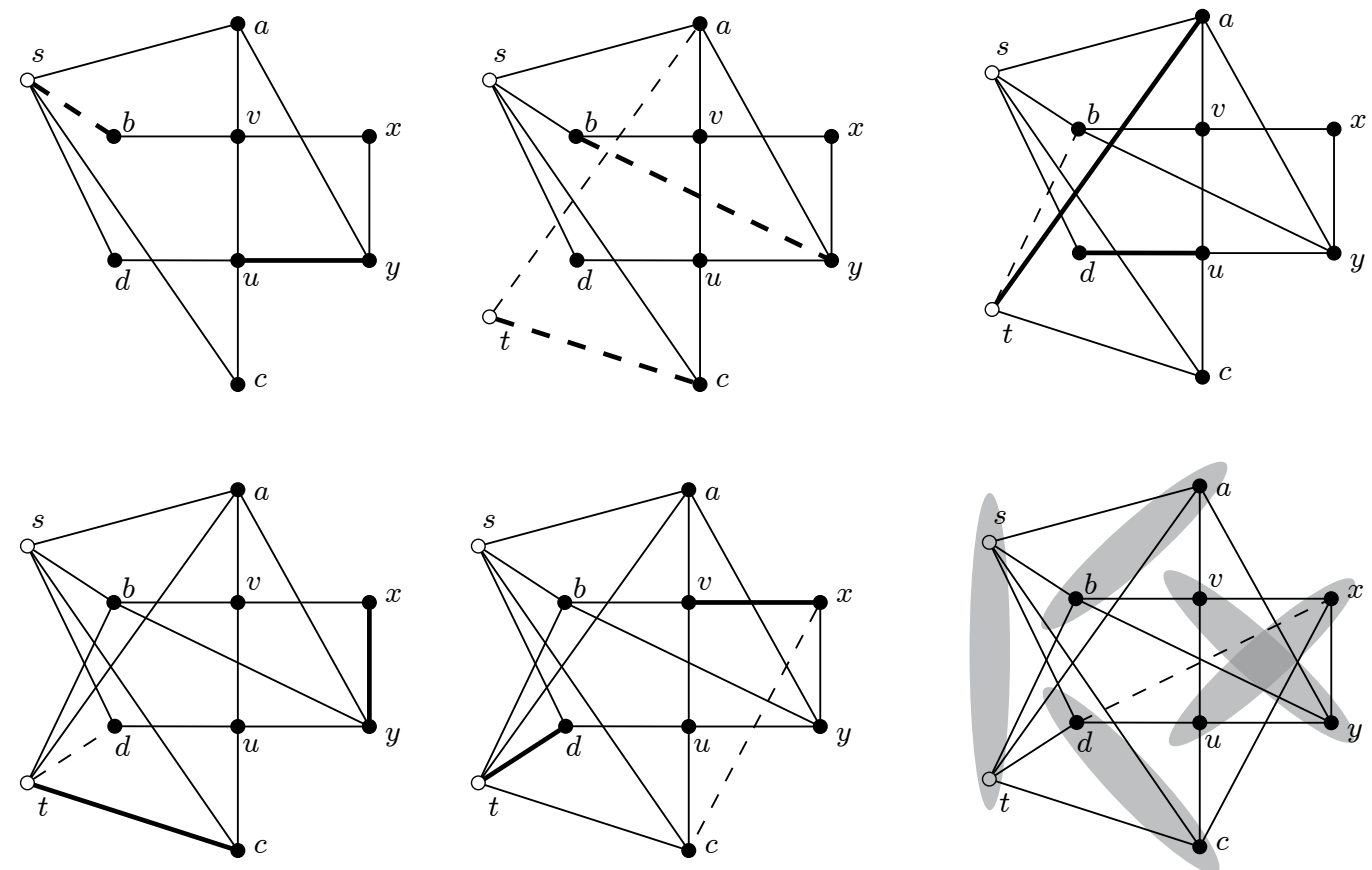

Figure 10: An illustration for Case 2.2; dashed edges indicate newly "found" edges and thick edges indicate considered pair in each step.

[2] A. Brandstädt, E. Eschen, and R. Sritharan, The induced matching and chain subgraph cover problems for convex bipartite graphs, Theoret. Comput. Sci. 381 (2007), no. 1-3, 260-265. 


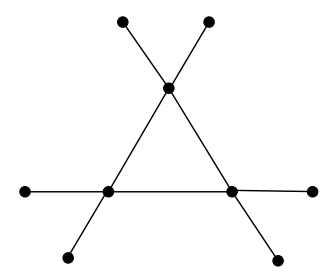

Figure 11: A tight example to Theorem 1.

[3] A. Brandstädt and C. T. Hoàng, Maximum induced matchings for chordal graphs in linear time, Algorithmica 52 (2008), no. 4, 440-447.

[4] K. Cameron, Induced matchings, Discrete Appl. Math. 24 (1989), no. 1-3, 97-102.

[5] K. Cameron, Induced matchings in intersection graphs. Discrete Math. 278 (2004), no. $1-3,1-9$.

[6] K. Cameron, R. Sritharan, and Y. Tang, Finding a maximum induced matching in weakly chordal graphs, Discrete Math. 266 (2003), no. 1-3, 133-142.

[7] J.-M. Chang, Induced matchings in asteroidal triple-free graphs, Discrete Appl. Math. 132 (2003), no. 1-3, 67-78.

[8] K. K. Dabrowski, M. Demange, and V. V. Lozin, New results on maximum induced matchings in bipartite graphs and beyond, Theoret. Comput. Sci. 478 (2013), 33-40.

[9] W. Duckworth, D. F. Manlove, and M. Zito, On the approximability of the maximum induced matching problem, J. Discrete Algorithms 3 (2005), no. 1, 79-91.

[10] J. Edmonds, Paths, trees, and flowers, Canad. J. Math. 17 (1965) 449-467.

[11] R. J. Faudree, R. H. Schelp, A. Gyárfás, and Zs. Tuza, The strong chromatic index of graphs. Ars Combin. 29 (1990), B, 205-211.

[12] M. C. Golumbic, and R. C. Laskar, Irredundancy in circular arc graphs, Discrete Appl. Math. 44 (1993), no. 1-3, 79-89.

[13] M. C. Golumbic and M. Lewenstein, New results on induced matchings, Discrete Appl. Math. 101 (2000), no. 1-3, 157-165.

[14] P. Horák, H. Qing, and W. T. Trotter, Induced matchings in cubic graphs, J. Graph Theory 17 (1993), no. 2, 151-160.

[15] F. Joos, Induced matchings in graphs of bounded maximum degree, arXiv:1406.2440, June 2014.

[16] F. Joos, D. Rautenbach, and T. Sasse, Induced matchings in subcubic graphs, SIAM J. Discrete Math. 28 (2014), no. 1, 468-473.

[17] D. Kobler and U. Rotics, Finding maximum induced matchings in subclasses of clawfree and $P_{5}$-free graphs, and in graphs with matching and induced matching of equal maximum size, Algorithmica 37 (2003), no. 4, 327-346. 
[18] R. J. Kang, M. Mnich, and T. Müller, Induced matchings in subcubic planar graphs SIAM J. Discrete Math. 26 (2012), no. 3, 1383-1411.

[19] V. V. Lozin On maximum induced matchings in bipartite graphs, Inform. Process. Lett. 81 (2002), no. 1, 7-11.

[20] M. Molloy and B. Reed, A bound on the strong chromatic index of a graph, J. Combin. Theory Ser. B 69 (1997), no. 2, 103-109.

[21] L. J. Stockmeyer and V. V. Vazirani, NP-completeness of some generalizations of the maximum matching problem, Inform. Process. Lett. 15 (1982), no. 1, 14-19.

[22] M. Zito, Induced matchings in regular graphs and trees, Lecture Notes in Comput. Sci., 1665, Springer, Berlin, 1999. 